\title{
The use of rapid dengue diagnostic tests in a routine clinical setting in a dengue-endemic area of Colombia
}

\author{
Lyda Osorio $/^{1 /+}$, Marcela Uribe 1 , Gloria Ines Ardila ${ }^{2}$, Yaneth Orejuela ${ }^{2}$, \\ Margarita Velasco $^{3,4}$, Anilza Bonelo ${ }^{3}$, Beatriz Parra ${ }^{5}$
}

\begin{abstract}
${ }^{1}$ Grupo de Investigación en Epidemiología y Salud Poblacional, Escuela de Salud Pública, Universidad del Valle, Cali, Colombia ${ }^{2}$ Laboratorio Especializado ${ }^{3}$ Grupo de Investigación e Evaluación de Servicios de Salud, Caja de Compensación Familiar del Valle del Cauca, Cali, Colombia ${ }^{4}$ Programa de Medicina, Facultad de Ciencias de la Salud, Universidad Libre, Cali, Colombia ${ }^{5}$ Grupo de Virus Emergentes y Enfermedad, Departamento de Microbiología, Universidad del Valle, Cali, Colombia
\end{abstract}

There is insufficient evidence of the usefulness of dengue diagnostic tests under routine conditions. We sought to analyse how physicians are using dengue diagnostics to inform research and development. Subjects attending 14 health institutions in an endemic area of Colombia with either a clinical diagnosis of dengue or for whom a dengue test was ordered were included in the study. Patterns of test-use are described herein. Factors associated with the ordering of dengue diagnostic tests were identified using contingency tables, nonparametric tests and logistic regression. A total of 778 subjects were diagnosed with dengue by the treating physician, of whom 386 (49.5\%) were tested for dengue. Another 491 dengue tests were ordered in subjects whose primary diagnosis was not dengue. Severe dengue classification [odds ratio (OR) 2.2; 95\% confidence interval (CI) 1.1-4.5], emergency consultation (OR 1.9; 95\% CI 1.4-2.5) and month of the year (OR 3.1; 95\% CI 1.7-5.5) were independently associated with ordering of dengue tests. Dengue tests were used both to rule in and rule out diagnosis. The latter use is not justified by the sensitivity of current rapid dengue diagnostic tests. Ordering of dengue tests appear to depend on a combination of factors, including physician and institutional preferences, as well as other patient and epidemiological factors.

Key words: dengue - diagnosis - health services - decision making

Dengue is an international public health emergency due to its rapid case number increase and fatality rate (Guzman et al. 2010). It is caused by one of four serotypes within the genus Flavivirus and is transmitted to humans by Aedes mosquitoes. Although it is present in most tropical and subtropical regions, the highest risk areas are in the Americas and Asia (Bhatt et al. 2013). The clinical presentation of dengue varies with age and immunological status and ranges from asymptomatic to severe and fatal infections. However, the factors associated with disease severity are not yet clearly understood. Abdominal pain or tenderness, persistent vomiting, clinical fluid accumulation, mucosal bleeding, lethargy, restlessness, liver enlargement $>2 \mathrm{~cm}$ and an increase in haematocrit concurrent with a rapid decrease in platelet count have been proposed as warning signs of disease progression to help improve case management (Alexander et al. 2011). Disease is considered severe in the presence of severe plasma

doi: 10.1590/0074-02760140359

Financial support: UniValle, COMFANDI

This study was partially funded by Standard Diagnostics Inc/Alere. However, the diagnostic test manufacturers do not have a role, either directly or through a third party, in the gathering or analysis of data, in the preparation or decision to submit the results for publication.

+ Corresponding author: 1ydaloso@gmail.com

Received 30 September 2014

Accepted 1 April 2015 leakage with shock and/or fluid accumulation with respiratory distress, severe bleeding or severe organ impairment (Alexander et al. 2011). It is expected that, based on these definitions, clinicians will be able to classify subjects as having dengue, with or without warning signs of severe dengue and treat them according to international guidelines (WHO/TDR 2012). There is not a specific antiviral treatment for dengue and hence, case management comprises adequate fluid support, rest, paracetamol and close monitoring until recovery (WHO/TDR 2012).

Dengue cases are confirmed by virus isolation, antigen or RNA detection, seroconversion or a fourfold increase in specific IgM or IgG titres (Kao et al. 2005). Several dengue diagnostic assays are available, but they are used mainly for research or surveillance due to the infrastructure they require, including a prolonged testing period, relatively high cost and the need for patient follow-up (Kao et al. 2005). There are commercially available rapid dengue diagnostic tests that are more suitable for routine use in health care settings (Blacksell 2012). However, laboratory diagnosis of dengue is not necessary for clinical management except in atypical cases or when ruling out differential diagnoses (WHO/ TDR 2012). In Colombia, the national guidelines stipulate the use of dengue diagnostic tests for surveillance purposes only (MPS/INS 2010). Despite this, rapid dengue diagnostic tests are frequently used within the country, perhaps due to the difficulty of diagnosis. Dengue diagnosis, under routine clinical care, is challenging because the typical clinical and laboratory characteristics of dengue in its febrile phase (temperature $\geq$ $38.5^{\circ} \mathrm{C}$ plus headache, vomiting, myalgia, joint pain and 
sometimes macular rash, haemorrhagic manifestations, thrombocytopaenia, leukopaenia and elevation of hepatic aminotransferase levels) or critical phase (increasing haemoconcentration, hypoproteinaemia, haemorrhagic manifestations, pleural effusion, ascites, narrowing of the pulse pressure, liver failure, myocarditis, encephalopathy, thrombocytopaenia, increase in the activated partial-thromboplastin time and decrease in fibrinogen levels) overlap with other diseases prevalent in the same endemic regions (Simmons et al. 2012). The importance of considering clinicians in the development and implementation of diagnostic tests has been highlighted, as they are the most knowledgeable concerning the many contributions of new technologies to health care (Feinstein 2002). Here, we sought to analyse how dengue rapid diagnostic tests (RDTs) are been routinely used in health care settings in endemic areas to inform research and development and health services.

\section{SUBJECTS, MATERIALS AND METHODS}

Study design and population - A prospective study was conducted from March-December 2012 at 14 health care institutions in Cali, Colombia. This city is one of the three largest in Colombia with a total population of 2,294,653 inhabitants. Dengue is considered hyperendemic due to the circulation of all four dengue serotypes. In 2010, one of the largest dengue epidemics hit the area with 11,047 cases, 5.7\% severe dengue and 16 attributable deaths. The 14 health care institutions included in the study were selected as they represent different levels of care (primary, secondary and tertiary) and have permanent access to rapid dengue diagnostic tests. Personnel at the participating institutions had been trained in dengue diagnosis and treatment in 2010 and 2011, therefore, the level of knowledge of dengue among physicians was considered high. Exhaustive sampling in the participating institutions was performed by including all subjects (regardless of age, sex or signs and symptoms) attending any of the 14 health care centres who were seen by a physician and were clinically diagnosed with dengue or for whom a dengue test was ordered during the study period. The study was approved by the Ethical Review Board of University of Valle and Family Compensation Fund of Valle del Cauca.

Rapid dengue diagnostic tests - Rapid dengue diagnostics were routinely available to all 14 institutions at a central lab. In August 2012, the largest institution (named here as A) was moved to a new building with its own lab and could perform dengue tests locally. At all 14 institutions, dengue diagnostics could be ordered at the physicians discretion at any time and results were made available through the computerised central clinical record system. During the study, the rapid dengue diagnostic test for the simultaneous detection of dengue-specific IgM and IgG (Standard Diagnostics Inc) was available at the laboratory facilities. The SD BIOLINE Dengue Duo kits (Standard Diagnostics Inc) that simultaneously detect IgM, IgG and NS1 were only occasionally available. Samples were processed by experienced laboratory technicians following the manufacturer's instructions. The study personnel did not have any direct or indirect contact with physicians to avoid any potential bias.
Data collection and quality control - Dengue cases were identified from clinical records with a diagnosis of dengue according to the codes of the International Classification of Diseases version 2010 (ICD-10) and from the forms used to notify the national surveillance system, which is compulsory (WHO 2010). Information on patient identification and demographics, health care institution, date and ward in which the subject was seen by the physician was provided by the centralised statistics department of the health care institutions. Data on all subjects for whom a dengue diagnostic test was ordered and the results of those tests were provided by the computerised laboratory information system of the central laboratory. Quality control of the data was performed by verifying respective patient identification numbers present in both databases. Indirect quality control of the technician in charge of dengue at the central lab was performed at the beginning of the study with three blind samples prepared by the research team. The results of these three samples remained consistent and further training was not considered necessary.

Statistical analysis - Clinical, laboratory and surveillance databases were merged and exported to Stata 10 (Stata Corp LP, USA) for data analysis. A descriptive analysis was conducted to identify the relative frequencies of the following: severity of dengue, cases in which a diagnostic test was ordered and IgM positivity index. The latter was defined as the percentage of IgM positive results out of the total IgM performed. Dengue cases were considered to be clinically diagnosed if one of ICD10 codes for dengue, A90 or A91, were reported in the clinical record. The dates when subjects were clinically diagnosed and tested for dengue were compared to ensure the clinical diagnosis preceded the lab test. When these dates were the same, the first reported diagnosis on the date was considered. Factors associated with ordering a dengue test in subjects with a clinical diagnosis of dengue were identified using contingency tables with the corresponding odds ratio (OR) and $95 \%$ confidence interval (CI) and chi-squared test or the Fisher exact test when necessary. For quantitative variables, nonparametric tests were used. Multivariate logistic regression models were fitted to estimated adjusted OR with their corresponding $95 \%$ CIs. A p-value $<0.05$ was considered statistically significant. Repeated dengue tests and those performed in subjects without an ICD-10 for dengue in their clinical records were analysed separately. It was assumed that the subjects clinical diagnosis was other than dengue when there were not matches between the lab database and both the dengue clinical and surveillance databases. For subjects for whom a dengue test was ordered, but whose clinical diagnosis and tests results were other than dengue, a descriptive analysis was performed.

\section{RESULTS}

A total of 778 subjects had a clinical diagnosis of dengue, of whom $39(5 \%)$ were classified as having severe dengue; $465(59.8 \%)$ were men and $275(35.4 \%)$ were zero-14 years old. The majority of cases (51.9\%) were reported by two (A and M) of the 14 institutions. Institution A (26.6\% of cases), a referral institution, was classified as offering 
TABLE I

Characteristics of subjects with clinical diagnosis of dengue

\begin{tabular}{|c|c|c|c|c|c|}
\hline \multirow[b]{3}{*}{ Characteristic } & \multicolumn{3}{|c|}{ Dengue cases } & \multirow[b]{3}{*}{ OR $(95 \% \mathrm{CI})$} & \multirow[b]{3}{*}{$\mathrm{p}$} \\
\hline & \multirow{2}{*}{$\begin{array}{c}\text { Total } \\
(\mathrm{n}=778) \\
\mathrm{n}(\%)\end{array}$} & $\begin{array}{c}\text { Nonsevere } \\
(\mathrm{n}=739 ; 95 \%)\end{array}$ & $\begin{array}{c}\text { Severe } \\
(\mathrm{n}=39 ; 5 \%)\end{array}$ & & \\
\hline & & n $(\%)$ & n $(\%)$ & & \\
\hline \multicolumn{6}{|l|}{ Sex } \\
\hline Male & $465(59.8)$ & $445(60.2)$ & $20(51.3)$ & - & 0.2 \\
\hline Female & $313(40.2)$ & $294(39.8)$ & $19(48.7)$ & $1.4(0.7-2.7)$ & \\
\hline \multicolumn{6}{|l|}{ Years of age } \\
\hline $\begin{array}{l}\text { Median } \\
\text { (range in years) }\end{array}$ & $20.8(0.6-91.8)$ & $20.9(0.6-91.8)$ & $19.4(0.6-60.3)$ & - & 0.9 \\
\hline $0-4.9$ & $72(9.3)$ & $68(9.2)$ & $4(10.3)$ & 1 & \\
\hline $5-14.9$ & $203(26.1)$ & $193(26.1)$ & $10(25.6)$ & $0.8(0.2-3)$ & 0.8 \\
\hline $15-44.9$ & $411(52.8)$ & $392(53.1)$ & $19(48.7)$ & $0.8(0.2-2.5)$ & 0.7 \\
\hline $45-64.9$ & $74(9.5)$ & $68(9.2)$ & $6(15.4)$ & $1.5(0.4-5.6)$ & 0.5 \\
\hline$\geq 65$ & $18(2.3)$ & $18(2.4)$ & $0(0)$ & 0 & 0.3 \\
\hline \multicolumn{6}{|l|}{ Institution } \\
\hline A & 207 (26.6) & $199(27)$ & $8(20.5)$ & 1 & 0.6 \\
\hline $\mathrm{B}$ & $6(0.7)$ & $6(0.8)$ & $0(0)$ & 0 & \\
\hline $\mathrm{C}$ & $73(9.4)$ & $70(9.5)$ & $3(7.7)$ & $1(0.2-4.1)$ & \\
\hline $\mathrm{D}$ & $40(5.1)$ & $38(5.1)$ & $2(5.1)$ & $1.3(0.2-6.4)$ & \\
\hline $\mathrm{E}$ & $26(3.3)$ & $25(3.4)$ & $1(2.5)$ & $1(0.1-8.3)$ & \\
\hline $\mathrm{F}$ & $23(3)$ & $23(3.1)$ & $0(0)$ & 0 & \\
\hline G & $81(10.5)$ & $79(10.7)$ & $2(5.1)$ & $0.6(0.1-3)$ & \\
\hline $\mathrm{H}$ & $40(5.1)$ & $35(4.7)$ & $5(12.8)$ & $3.5(1-11.6)$ & \\
\hline I & $23(3)$ & $22(39)$ & $1(2.5)$ & $1.1(0.1-9.5)$ & \\
\hline $\mathrm{J}$ & $11(1.4)$ & $10(1.3)$ & $1(2.5)$ & $2.4(0.2-22)$ & \\
\hline $\mathrm{K}$ & $36(4.6)$ & $34(4.6)$ & $2(5.1)$ & $1.4(0.3-7.2)$ & \\
\hline $\mathrm{L}$ & $1(0.1)$ & $1(0.1)$ & $0(0)$ & 0 & \\
\hline M & $197(25.3)$ & $183(24.7)$ & $14(36)$ & $2(0.7-4.6)$ & \\
\hline $\mathrm{N}$ & $14(1.8)$ & $14(1.9)$ & $0(0)$ & 0 & \\
\hline \multicolumn{6}{|l|}{ Ward } \\
\hline Outpatient & $408(52.4)$ & $389(52.6)$ & $19(48.3)$ & 1 & \\
\hline Emergency & $370(47.6)$ & $350(47.4)$ & $20(51.3)$ & $1.1(0.6-2.2)$ & 0.6 \\
\hline \multicolumn{6}{|l|}{ Month } \\
\hline March & $127(16.3)$ & $120(16.2)$ & 7 (18) & 1 & 0.1 \\
\hline April & $84(10.8)$ & $79(10.7)$ & $5(12.8)$ & $1.1(0.3-3.5)$ & \\
\hline May & $57(7.3)$ & $55(7.4)$ & $2(5.1)$ & $0.6(0.1-3.1)$ & \\
\hline June & $57(7.3)$ & $55(7.4)$ & $2(5.1)$ & $0.6(0.1-3.1)$ & \\
\hline July & $56(7.2)$ & $53(7.1)$ & $3(7.7)$ & $0.9(0.2-4)$ & \\
\hline August & $61(7.8)$ & $61(7.8)$ & $0(0)$ & 0 & \\
\hline September & $72(9.2)$ & $72(9.2)$ & $9(23.1)$ & $2.4(0.8-7)$ & \\
\hline October & $80(10.3)$ & $80(10.3)$ & $5(12.8)$ & $1.1(0.3-3.7)$ & \\
\hline November & $61(7.8)$ & $61(7.8)$ & $2(5.1)$ & $0.6(0.1-3)$ & \\
\hline December & $123(15.8)$ & $123(15.8)$ & $4(10.3)$ & $0.6(0.1-2)$ & \\
\hline \multicolumn{6}{|l|}{ Total dengue tests } \\
\hline 0 & $393(50.5)$ & $380(51.4)$ & $13(33.3)$ & 1 & \\
\hline 1 & $320(41.1)$ & $297(40.2)$ & $23(59)$ & $2.2(1.1-4.5)$ & 0.01 \\
\hline 2 & $53(6.8)$ & $50(6.8)$ & $3(7.7)$ & $1.7(0.5-6.3)$ & 0.4 \\
\hline 3 & $11(1.4)$ & $11(1.5)$ & $0(0)$ & 0 & 0.5 \\
\hline 5 & $1(0.2)$ & $1(0.1)$ & $0(0)$ & 0 & 0.8 \\
\hline
\end{tabular}

CI: confidence interval; OR: odds ratio. 
a tertiary level of care, while the institution M $(25.3 \%$ of cases) was classified as offering secondary level of care. Subjects with severe cases were more frequently female, between 45-64.9 years old and seen at the emergency wards with the highest frequency in September. However, there were no statistically significant differences between severe and nonsevere cases regarding sex, age, institution, month or emergency consultation (Table I).

At least one dengue diagnostic test was ordered in $386(49.5 \%)$ subjects with slight monthly variations (Figure). Age and sex of subjects were not associated with the ordering of a dengue test, but with severity of disease, emergency consultation, institution and month. All of these factors remained independently associated in the multivariate model; however, institution was withdrawn from the model as a correlation with emergency consultation existed (Table II).

The IgM positivity index was $30 \%(114 / 386)$, but it varied monthly reaching up to $58 \%$ in November (Figure). The IgM positivity index was twice as high in severe dengue cases $(46.1 \%$-12/26) as in nonsevere dengue cases $(28.3 \%-102 / 360)$ cases $(p=0.05)$. During the study period, 491 dengue tests were ordered for subjects who did not have an explicit clinical diagnosis of dengue in their clinical record, but another diagnosis (such as upper and lower respiratory tract infections, unspecified viral disease, meningitis, unspecified thrombocytopenia, human immunodeficiency virus, diarrhoea, malaria, leptospirosis, among others). In this group, the IgM positivity index was 3.6\% (18/491).

More than one dengue test was ordered in 65 subjects: two tests in 53, three in 11 and five in one person. There was no difference in the frequency of repeated dengue tests between severe (3-7.7\%) and nonsevere dengue cases (62-8.4\%). Of the 58 subjects with negative IgM or IgG results in the first test, IgM seroconversion was observed in 15 subjects in the second test and three subjects in the third test. Similar results were found with $\mathrm{IgG}$, with seroconversion observed in 15 subjects in the second test and three subjects in the third test. No seroconversion was observed in subsequent tests. Only two subjects were tested twice with NS1 and negative results occurred in both cases. Changes from positive to negative $\operatorname{IgM}$ or IgG results were observed in two subjects.

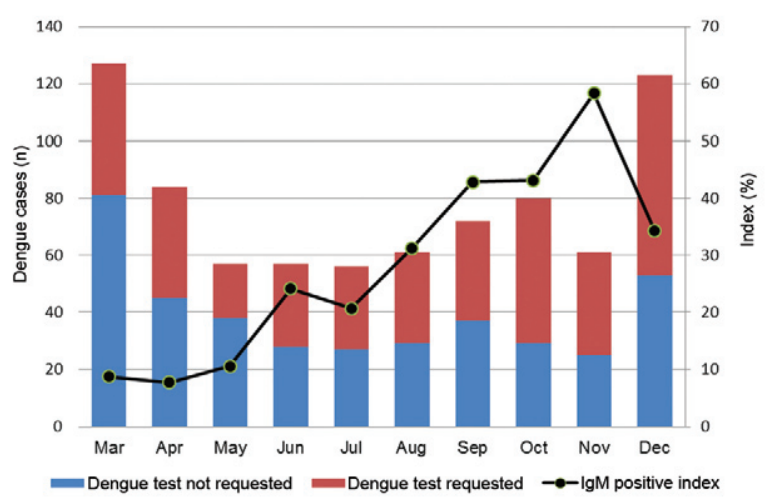

Monthly trends in number of dengue rapid diagnostic tests requested and IgM positive results.

\section{DISCUSSION}

In the present study, we analyse the use of rapid dengue diagnostic tests under routine conditions in health care settings in an endemic area of Colombia. An emerging pattern in this study was the use of RDTs to either rule in or rule out dengue as a differential diagnosis. Half of the subjects with a clinical diagnosis of dengue had a dengue test ordered (i.e., a test used to rule in dengue) but subjects with other clinical diagnosis were also tested for dengue (i.e., a test use to rule out dengue). The use of dengue tests to confirm dengue diagnosis is supported by the relatively high specificity of the currently available tests, which is required to rule in a diagnosis. The reported specificity of the two tests used in the study sites, namely SD BIOLINE Dengue IgM/IgG and SD BIOLINE Dengue Duo NS1, IgM/IgG RDT, ranges from $86.8-92.3 \%$ and from $83.9-100 \%$, respectively (WHO/ TDR 2009, Tricou et al. 2010, Blacksell et al. 2011, Gan et al. 2014). However, to date, there is no evidence that the dengue test results influence the physicians behaviour or impact the prognosis of a subject for whom a clinical diagnosis of dengue was already made (Andries et al. 2012). Hence, it is necessary to further assess the impact and cost-effectiveness of implementing rapid dengue diagnostics tests in real-life settings, accounting for evidence-based decisions. Contrastingly, high sensitivity of the test is required to rule out a diagnosis. Rapid dengue diagnostic tests have shown to have a large variation in sensitivities ranging from $47-79.2 \%$ for $\operatorname{IgM} / \mathrm{IgG}$ and from $78.4-93.9 \%$ for $\mathrm{NS} 1 / \mathrm{IgM} / \mathrm{IgG}$ (WHO/TDR 2009, Osorio et al. 2010, Gan et al. 2014). Consequently, they are not suitable for ruling out dengue or for screening purposes. Understanding why physicians decide to order dengue tests is therefore worth exploring, while using their input to improve current rapid diagnostic methods.

The results show that severe dengue presentation, emergency consultation and month were all independently associated with the ordering of dengue diagnostic tests. Several explanations could be considered for these findings. First, due to the potential fatal consequences of misdiagnosis in a severe case, clinicians may consider confirmation of these cases to be more relevant than in the nonsevere cases or seek assurances for themselves and the patient/patient's family in the validity of a positive result when ordering a dengue diagnostic test. The need for assurance can be interpreted as defensive in case of possible litigation or ones own expectations of selfefficacy (i.e., performing as expected), while the need to reassure a worried patient/patient's family can be either real or perceived due to social pressure (van der Weijden et al. 2002). Secondly, RDTs are expected to be used at bedside; hence in the context of emergency care, rapid dengue diagnostic tests could be thought of as an accessible tool to speed up the differential diagnosis process and corresponding management (Peeling et al. 2010). Alternatively, this factor may be explained by the inclusion of emergency departments located in the secondary and tertiary levels of care where it is known that diagnostic tests are more commonly used to rule in a disease. In contrast, diagnostics are used more often to rule out a condition or to determine the need for referral in primary care centres 
TABLE II

Factors associated with requesting a dengue rapid diagnostic test in subjects with clinical diagnosis of dengue

\begin{tabular}{|c|c|c|c|c|c|c|}
\hline \multirow[b]{2}{*}{ Characteristic } & \multicolumn{2}{|c|}{ Dengue test requested } & \multirow[b]{2}{*}{ OR $(95 \%$ CI $)$} & \multirow[b]{2}{*}{$\mathrm{p}$} & \multirow[b]{2}{*}{$\begin{array}{c}\text { Adjusted } \\
\text { OR }(95 \% \text { CI })\end{array}$} & \multirow[b]{2}{*}{$\mathrm{p}$} \\
\hline & $\begin{array}{c}\text { Yes } \\
(\mathrm{n}=386) \\
\mathrm{n}(\%)\end{array}$ & $\begin{array}{c}\text { No } \\
(\mathrm{n}=392) \\
\mathrm{n}(\%)\end{array}$ & & & & \\
\hline \multicolumn{7}{|l|}{ Sex } \\
\hline Male & $236(61.1)$ & $229(58.4)$ & 1 & & - & \\
\hline Female & $150(38.9)$ & $163(41.6)$ & $0.8(0.7-1.2)$ & 0.4 & - & \\
\hline \multicolumn{7}{|l|}{ Years of age } \\
\hline $\begin{array}{l}\text { Median } \\
\text { (range in years) }\end{array}$ & $20.8(0.6-79)$ & $20.9(0.3-91.8)$ & - & 0.3 & - & \\
\hline $0-4$ & $31(8)$ & $41(10.4)$ & 1 & & - & \\
\hline $5-14$ & $104(27)$ & $100(25.3)$ & $1.4(0.8-2.3)$ & 0.2 & - & \\
\hline $15-44$ & $206(53.4)$ & $205(52.3)$ & $1.3(0.8-2.2)$ & 0.2 & - & \\
\hline $45-64$ & $39(10.1)$ & $35(9)$ & $1.5(0.7-2.8)$ & 0.2 & - & \\
\hline$\geq 65$ & $6(1.5)$ & $12(3)$ & $0.6(0.2-2)$ & 0.4 & - & \\
\hline \multicolumn{7}{|l|}{ Dengue classification } \\
\hline Nonsevere & $360(93.2)$ & $379(96.7)$ & 1 & & - & \\
\hline Severe & $26(6.8)$ & $13(3.3)$ & $2.1(1-4.2)$ & 0.03 & $2.2(1.1-4.5)$ & 0.02 \\
\hline \multicolumn{7}{|c|}{ Institution level of care } \\
\hline Primary & $147(38.1)$ & $221(56.4)$ & 1 & & $a$ & \\
\hline Secondary & $93(24.1)$ & $104(26.5)$ & $1.3(0.9-1.9)$ & 0.09 & - & \\
\hline Tertiary & $146(37.8)$ & $67(17.1)$ & $3.3(2.2-4.7)$ & $<0.001$ & - & \\
\hline \multicolumn{7}{|l|}{ Ward } \\
\hline Outpatient & $171(44.3)$ & $237(60.5)$ & - & & - & \\
\hline Emergency & $215(55.7)$ & $155(39.5)$ & $2(1.4-2.5)$ & $<0.001$ & $1.9(1.4-2.5)$ & $<0.001$ \\
\hline \multicolumn{7}{|l|}{ Month } \\
\hline March & $46(12)$ & $81(20.7)$ & 1 & & 1 & \\
\hline April & $39(10.1)$ & 45 (11.5) & $1.5(0.8-2.6)$ & 0.1 & $1.4(0.7-2.4)$ & 0.2 \\
\hline May & $19(5)$ & $38(9.7)$ & $0.8(0.4-1.7)$ & 0.7 & $0.7(0.4-1.5)$ & 0.5 \\
\hline June & $29(7.5)$ & $28(7.1)$ & $1.8(0.9-3.4)$ & 0.06 & $1.7(0.9-3.3)$ & 0.08 \\
\hline July & $29(7.5)$ & 27 (6.9) & $1.9(1-3.5)$ & 0.05 & $1.6(0.8-3.1)$ & 0.1 \\
\hline August & $32(8.3)$ & $29(7.4)$ & $1.9(1-3.6)$ & 0.03 & $1.9(1-3.6)$ & 0.04 \\
\hline September & $35(9)$ & $37(9.4)$ & $1.6(0.9-3)$ & 0.08 & $1.5(0.8-2.7)$ & 0.2 \\
\hline October & $51(13.2)$ & $29(7.4)$ & $3(1.7-5.5)$ & $<0.001$ & $3.1(1.7-5.5)$ & $<0.001$ \\
\hline November & $36(9.3)$ & $25(6.4)$ & $2.5(1.3-4.7)$ & 0.004 & $2.3(1.2-4.4)$ & 0.008 \\
\hline December & $70(18.1)$ & $53(13.5)$ & $2.3(1.4-3.8)$ & 0.001 & $2(1.2-3.4)$ & 0.006 \\
\hline
\end{tabular}

$a$ : withdrawn from the model because of colinearity with institution; CI: confidence interval; OR: odds ratio.

(Whiting et al. 2007). Finally, the association between ordering rapid dengue diagnostic tests with the month of the year may suggest that clinicians use diagnostic tests to detect outbreaks of febrile diseases, which is relevant to countries such as Colombia, where there is not a distinctive seasonality of dengue incidence. Of the subjects meeting clinical criteria for dengue, the probability of in fact having dengue is lower in nonepidemic periods than during epidemics. Hence, laboratory diagnosis may help physicians to adjust the predictive values of the clinical definition. This epidemiological reason for ordering dengue tests could be useful in routine care by adjusting of the predictive values of the clinical definitions of disease in subsequent patients or to increase awareness of diseases. Hence, improved laboratory based surveillance that informs clinicians of dengue outbreak at the local level have been proposed to assist them (Lorenzi et al. 2013). This epidemiological use of health technology is rarely recognised and could be added to the five categories of factors (diagnostic, therapeutic and prognostic, patientrelated, doctor-related and policy and organisation-related) that influence ordering of diagnostic tests previously proposed (Whiting et al. 2007).

During field work, a dengue epidemic was not declared in the study site, but the IgM positivity steadily increased reaching $58 \%$ in November. However, this 
increase in IgM positivity preceded an epidemic that was declared early in 2013 in the study site. This finding further supports the monitoring of IgM positivity as a potential tool for early outbreak detection (Hati 2009). A cut-off point above which an IgM positive index suggests a dengue epidemic will need to be validated. NS1 positive index could also be explored. There were too few positive NS1 samples in the present study to assess this marker. Performing more than one RDT in the same subject was useful to identify dengue cases through seroconversion of $\operatorname{IgM}$ and $\operatorname{IgG}$ specific antibodies, but further studies are required to assess the cost-effectiveness of this practice in the routine care.

There are several limitations to the present study. Reasons for ordering diagnostic tests are multiple and complex. While our approach was eminently quantitative and allowed us to identify factors associated with the use of rapid dengue diagnostic tests, a qualitative approach would be complementary to identify other factors that are not measurable by quantitative methods. Dengue diagnosis and classification was based on the ICD-10 available in the computerised system, which does not include the most recent definitions proposed by World Health Organization (WHO/TDR 2012). Hence, it was not possible to explore the patterns of use of RDTs in cases classified as dengue with warning signs. Time-series analysis was not performed because the number of monthly observations $(n=10)$ was considered insufficient to yield reliable results. Finally, detailed information of signs, symptoms and onset of fever were not available and could not be explored for their association with ordering diagnostic tests. Particularly, the latter is expected to be critical to the use of dengue RDT as the sensitivity of these tests varies with time. After data analysis, we were able to retrieve information on date of disease onset on 296 subjects from the dengue surveillance database at the local public health office. Dengue RDTs were performed in 109 of these subjects with a median of four (range 0-21) days of symptoms compared to five (range 0-180) days in subjects who were not tested. Surveillance records also contain detailed information of signs and symptoms, but neither this nor dates of onset of disease were used in the analysis because this data was not validated and was unavailable for most study subjects.

In conclusion, rapid dengue diagnostic tests are been used to both rule in and rule out disease. The latter highlights the need for improved sensitivity of currently available rapid dengue diagnostic tests. Further studies, which consider the influence of nonevidencebased reasons for using health technology, such as reassurance for clinicians or patients/families as well as epidemiological reasons, are required to assess the cost-effectiveness of implementing rapid dengue diagnostic tests in routine care in endemic areas.

\section{ACKNOWLEDGEMENTS}

To Dr Martha Lucia Ramos, from COMFANDI, for support to perform the study, and to Melissa Pelaez, Liliana Soto and Claudia Vidal, from UniValle, for their assistance.

\section{REFERENCES}

Alexander N, Balmaseda A, Coelho IC, Dimaano E, Hien TT, Hung NT, Jänisch T, Kroeger A, Lum LC, Martinez E, Siqueira JB, Thuy TT, Villalobos I, Villegas E, Wills B, World Health Organization (WHO-TDR) supported DENCO Study Group European Union 2011. Multicentre prospective study on dengue classification in four South-east Asian and three Latin American countries. Trop Med Int Health 16: 936-948.

Andries AC, Duong V, Ngan C, Ong S, Huy R, Sroin KK, Te V, Bunthin Y, Try PL, Buchy P 2012. Field evaluation and impact on clinical management of a rapid diagnostic kit that detects dengue NS1, IgM and IgG. PLoS Negl Trop Dis 6: e1993.

Bhatt S, Gething PW, Brady OJ, Messina JP, Farlow AW, Moyes CL, Drake JM, Brownstein JS, Hoen AG, Sankoh O, Myers MF, George DB, Jaenisch T, Wint GR, Simmons CP, Scott TW, Farrar JJ, Hay SI 2013. The global distribution and burden of dengue. Nature 496: 504-507.

Blacksell SD 2012. Commercial dengue rapid diagnostic tests for point-of-care application: recent evaluations and future needs? $J$ Biomed Biotechnol 2012: 151967.

Blacksell SD, Jarman RG, Bailey MS, Tanganuchitcharnchai A, Jenjaroen K, Gibbons RV, Paris DH, Premaratna R, de Silva HJ, Lalloo DG, Day NP 2011. Evaluation of six commercial point-of-care tests for diagnosis of acute dengue infections: the need for combining NS1 antigen and $\mathrm{IgM} / \mathrm{IgG}$ antibody detection to achieve acceptable levels of accuracy. Clin Vaccine Immunol 18: 2095-2101.

Feinstein AR 2002. Misguided efforts and future challenges for research on "diagnostic tests". J Epidemiol Community Health 56: 330-332.

Gan VC, Tan LK, Lye DC, Pok KY, Mok SQ, Chua RC, Leo YS, Ng LC 2014. Diagnosing dengue at the point-of-care: utility of a rapid combined diagnostic kit in Singapore. PLOS ONE 9: e90037.

Guzman MG, Halstead SB, Artsob H, Buchy P, Farrar J, Gubler DJ, Hunsperger E, Kroeger A, Margolis HS, Martínez E, Nathan MB, Pelegrino JL, Simmons C, Yoksan S, Peeling RW 2010. Dengue: a continuing global threat. Nat Rev Microbiol 8 (Suppl. 12): S7-S16.

Hati AK 2009. Dengue serosurveillance in Kolkata, facing an epidemic in West Bengal, India. J Vector Borne Dis 46: 197-204.

Kao CL, King CC, Chao DY, Wu HL, Chang GJ 2005. Laboratory diagnosis of dengue virus infection: current and future perspectives in clinical diagnosis and public health. $J$ Microbiol Immunol Infect 38: 5-16.

Lorenzi OD, Gregory CJ, Santiago LM, Acosta H, Galarza IE, Hunsperger E, Muñoz J, Bui DM, Oberste MS, Peñaranda S, GarcíaGubern C, Tomashek KM, Saint Luke's Acute Febrile Illness Investigation Team 2013. Acute febrile illness surveillance in a tertiary hospital emergency department: comparison of influenza and dengue virus infections. Am J Trop Med Hyg 88: 472-480.

MPS/INS/OPS - Ministerio de la Protección Social/Instituto Nacional de Salud/Organización Panamericana de la Salud 2010. Guía para la atención clínica integral del paciente con dengue, OPS/OMS, Bogotá, 45 pp.

Osorio L, Ramirez M, Bonelo A, Villar LA, Parra B 2010. Comparison of the diagnostic accuracy of commercial NS1-based diagnostic tests for early dengue infection. Virol $J$ 7: 361.

Peeling RW, Artsob H, Pelegrino JL, Buchy P, Cardosa MJ, Devi S, Enria DA, Farrar J, Gubler DJ, Guzman MG, Halstead SB, Hunsperger E, Kliks S, Margolis HS, Nathanson CM, Nguyen VC, 
Rizzo N, Vazquez S, Yoksan S 2010. Evaluation of diagnostic tests: dengue. Nat Rev Microbiol 8 (Suppl. 12): S30-S38.

Simmons CP, Farrar JJ, Nguyen V, Wills B 2012. Dengue. $N$ Engl J Med 366: 1423-1432.

Tricou V, Vu HT, Quynh NV, Nguyen CV, Tran HT, Farrar J, Wills B, Simmons CP 2010. Comparison of two dengue NS1 rapid tests for sensitivity, specificity and relationship to viraemia and antibody responses. BMC Infect Dis 10: 142.

van der Weijden T, van Bokhoven MA, Dinant GJ, van Hasselt CM, Grol RP 2002. Understanding laboratory testing in diagnostic uncertainty: a qualitative study in general practice. $\mathrm{Br} J \mathrm{Gen}$ Pract 52: 974-980.
Whiting P, Toerien M, de Salis I, Sterne JA, Dieppe P, Egger M, Fahey $\mathrm{T}$ 2007. A review identifies and classifies reasons for ordering diagnostic tests. J Clin Epidemiol 60: 981-989.

WHO - World Health Organization 2010. International Classification of Diseases version 2010. Available from: apps.who.int/classifications/icd10/browse/2010/en.

WHO/TDR - World Health Organization/Special Programme for Research and Training in Tropical Diseases 2009. Diagnostics evaluation Series 3, Evaluation of commercially available antidengue virus immunoglobulin tests, WHO, Geneva, 42 pp.

WHO/TDR - World Health Organization/Special Programme for Tropical Diseases Research 2012. Handbook for clinical management of dengue, WHO, Geneva, $123 \mathrm{pp}$. 